\title{
Alterações cardiovasculares de gatos submetidos à toracotomia intercostal, pré-medicados com associação de tramadol, butorfanol e atropina e anestesiados com propofol e halotano
}

\author{
Cardiovascular changes in cats submitted to intercostal thoracotomy, premedication with \\ association tramadol, butorphanol, atropine, anesthetised with propofol and halothane
}

\section{Juliana Tabarelli Brondani ${ }^{1}$ Cláudio Corrêa Natalini ${ }^{2}$ João Eduardo Wallau Schossler ${ }^{3}$ Saulo Tadeu Lemos Pinto Filho ${ }^{4}$ Adriana Paula Bertin ${ }^{5}$}

\section{RESUMO}

A toracotomia é um procedimento cirúrgico que produz estímulo doloroso intenso. $O$ objetivo deste estudo foi avaliar o efeito cardiovascular da associação tramadol, butorfanol e atropina na medicação pré-anestésica de gatos anestesiados com propofol e halotano. Doze animais, SRD, machos ou fêmeas, com peso médio de 2,7 $\pm 0,62 \mathrm{~kg}$ receberam como medicação pré-anestésica (MPA), a associação de tramadol (2,0mg $\left.\mathrm{kg}^{-1}\right)$, butorfanol $\left(0,4 \mathrm{mg} \mathrm{kg}^{-1}\right)$ e atropina $\left(0,044 \mathrm{mg} \mathrm{kg}^{-1}\right)$, via intramuscular. Trinta minutos após MPA, a indução foi realizada com propofol (5,0mg $\left.\mathrm{kg}^{-1}\right)$ por via intravenosa. $A$ manutenção anestésica foi obtida com halotano e oxigênio $100 \%$ sob ventilação artificial manual. Os gatos foram submetidos à toracotomia intercostal para implante de um segmento autólogo de pericárdio no diafragma. As variáveis avaliadas foram: freqüencia cardiaca (bpm), saturação de oxigênio da hemoglobina (\%), pressão arterial sistólica ( $\mathrm{mmHg}$ ) e vaporização de halotano (\%). As variáveis foram mensuradas 20 minutos após a MPA (TMPA), 10 minutos após indução e a cada 10 minutos até o final do procedimento cirúrgico (T10 a T100). Os dados obtidos foram analisados estatisticamente através de ANOVA e teste de Bonferroni $(p<0,05)$. Os resultados demonstraram redução na pressão arterial sistólica e freqüencia cardiaca, estatisticamente significativa $(p<0,05)$, porém, se mantiveram dentro da faixa de variação fisiológica para a espécie felina. Saturação de oxigênio da hemoglobina manteve-se ao redor de $100 \%$ em todos os tempos. A vaporização de halotano diminuiu significativamente do T30 ao T100, permancendo na maior parte do tempo cirúrgico abaixo da concentração alveolar mínima para gatos. Conclui-se que a associação tramadol (2,0mg $\left.\mathrm{kg}^{-1}\right)$, butorfanol $\left(0,4 \mathrm{mg} \mathrm{kg}^{-1}\right)$ e atropina $\left(0,044 \mathrm{mg} \mathrm{kg}^{-1}\right)$, via intramuscular, como pré-medicação, em gatos, anestesiados com propofol $\left(5,0 \mathrm{mg} \mathrm{kg}^{-1}\right)$ e halotano produz mínimas alterações cardiovasculares, não induz sedação e promove analgesia satisfatória para realização de toracotomia intercostal lateral.

Palavras-chave: gatos, toracotomia, tramadol, butorfanol.

\section{ABSTRACT}

Intercostal thoracotomy is a very painful procedure that deserves proper prevention and treatment. In this study we aimed to investigate the cardiovascular effect of the association of tramadol, butorphanol and atropine in the premedication of cats anesthetised with propofol and halothane. Twelve cats of mixed breed, female and male, with mean body weight of $2.7 \pm$ $0.62 \mathrm{~kg}$ were premedicated with $2.0 \mathrm{mg} \mathrm{kg}^{-1}$ tramadol and $0.4 \mathrm{mg}$

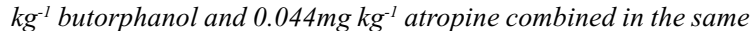
syringe intramuscularly administered. After 30 minutes of premedication, anesthetic induction was obtained with $5.0 \mathrm{mg} \mathrm{kg}^{-1}$ propofol intravenously. Anesthetic maintenance was done with halothane and $100 \%$ oxygen with manual artificial ventilation. All cats were submitted to lateral intercostal thoracotomy for an autogenic pericardium graft implantation in the diaphragm. Variables studied were heart rate ( $\mathrm{bpm}$ ), hemoglobin oxygen saturation (\%), systolic arterial pressure $(\mathrm{mmHg})$, and halothane vaporization (\%). Time for data collection were 20 minutes after premedication (TMPA), 10 minutes after induction and every 10 minutes up to the end of the surgical procedure (T10 to T100).

${ }^{1}$ Médico Veterinário, Mestrando do Programa de Pós-graduação em Medicina Veterinária, Universidade Federal de Santa Maria (UFSM). Endereço para correspondência: Hospital Veterinário/UFSM, Faixa de Camobi, Km 9, 97105-900, Santa Maria-RS. E-mail: jtbrondani@yahoo.com Autor para correspondência.

${ }^{2}$ Médico Veterinário, Professor Veterinary Anesthesiology and Pain Management, PhD, Louisiana State University, Baton Rouge -EUA.

${ }^{3}$ Médico Veterinário, Professor Adjunto, Doutor, UFSM.

${ }^{4}$ Médico Veterinário, Mestre em Cirurgia Veterinária, UFSM.

${ }^{5}$ Acadêmica do Curso de Medicina Veterinária, UFSM. 
Data were analyzed with ANOVA and Bonferroni's test $(p<0.05)$. Results demonstrated statistically significant reduction in arterial systolic pressure and heart rate, however, kept within the physiologic variation parameter to felines. Hemoglobin oxygen saturation was close to $100 \%$ in all times. Halothane vaporization decreased significantly from T30 to T100, staying for most of the surgical time below the minim alveolar concentration for cats. In conclusion the assotiation tramadol (2.0mg $\left.\mathrm{kg}^{-1}\right)$, butorphanol $\left(0.4 \mathrm{mg} \mathrm{kg}^{-1}\right)$ and atropine $\left(0.044 \mathrm{mg} \mathrm{kg}^{-1}\right)$ in the premedication of cats anesthetised with propofol (5.0mg $\left.\mathrm{kg}^{-1}\right)$ and halothane, intramuscularly, produces minimal changes cardiovasculares, don't produce sedation and promote analgesia satisfactory for lateral intercostal thoracotomy.

Key words: cats, thoracotomy, tramadol, butorphanol.

\section{INTRODUÇÃO}

A toracotomia é considerada um procedimento cirúrgico que produz dor moderada a severa, desta forma, devem-se selecionar analgésicos apropriados para controle da dor trans e pós-cirúrgica (JOHNSON, 1991; WERNER \& TABOADA, 1994).

Quando se antecipa que o procedimento cirúrgico produzirá dor moderada a severa, fármacos com propriedades analgésicas deverão ser administrados no período pré-operatório com o intuito de se produzir analgesia preventiva. A analgesia preventiva é mais eficaz, além de diminuir significativamente a dose dos fármacos anestésicos requeridos para indução e manutenção, permite que o animal seja mantido em um plano anestésico superficial, minimizando o grau de depressão cardiorrespiratória induzida pelos anestésicos (HELLYER \& GAYNOR, 1998).

O butorfanol é um opióide agonistaantagonista com alta afinidade por receptores kappa produzindo efeito agonista e moderada afinidade por receptores $m u$ com efeito antagonista competitivo (ORSINI, 1988). Desta forma, a ligação do butorfanol a receptores kappa produz variados graus de analgesia e sedação com mínima depressão cardiorrespiratória (WARNER, 1999).

O butorfanol é um analgésico amplamente utilizado em gatos para promover analgesia visceral e somática. Baixas doses $\left(0,2 \mathrm{mg} \mathrm{kg}^{-l}\right)$ produzem efetiva analgesia visceral em gatos por um período de até seis horas; doses maiores $\left(0,8 \mathrm{mg} \mathrm{kg}^{-1}\right)$ são eficientes para analgesia somática, porém por um período de até duas horas (WAGNER, 1999).

O tramadol é um analgésico de ação central que atua em receptores opióides, além de modificar a transmissão de impulsos da dor através da inibição da recaptação de monoaminas (LEE et al., 1993; SCOTT \& PERRY, 2000). O tramadol produz antinocicepção por dois mecanismos de ação diferentes e independentes, mas sinérgicos, age como agonista opióide $m u$ e inibe a recaptação neuronal de norepinefrina e serotonina (RAFFA et al.,1992; DAYER et al., 1997). Não se encontram na literatura referências sobre o uso de tramadol em gatos.

O objetivo deste estudo foi avaliar o efeito cardiovascular da associação tramadol, butorfanol e atropina na pré-medicação de gatos anestesiados com propofol e halotano e submetidos à toracotomia intercostal.

\section{MATERIAL E METODOS}

Foram utilizados 12 gatos adultos, 10 fêmeas e 2 machos, sem raça definida, com peso médio de 2,7 $\pm 0,62 \mathrm{~kg}$, provenientes do Biotério Central da UFSM, considerados hígidos após exame clínico completo. Os animais foram submetidos a jejum para dieta sólida de 12 horas e jejum líquido de quatro horas anteriores ao procedimento cirúrgico.

Os animais foram pré-medicados com associação de tramadol ${ }^{\mathrm{a}}\left(2,0 \mathrm{mg} \mathrm{kg}^{-1}\right)$, butorfanol ${ }^{\mathrm{b}}$

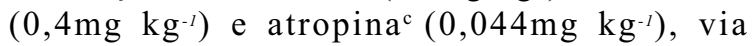
intramuscular. Após trinta minutos, foi adaptado na veia cefálica, um catéter $24 \mathrm{G}$, para administração do agente indutor (propofol) e de solução cloreto de sódio $0,9 \%$ na dose de $10 \mathrm{ml} \mathrm{kg}^{-l} \mathrm{~h}^{-1}$.

A indução anestésica foi realizada com propofol $^{\mathrm{d}}$ na dose de $5,0 \mathrm{mg} \mathrm{kg}{ }^{-1}$. Os animais foram intubados e mantidos com halotano e oxigênio $100 \%$ sob ventilação artificial manual com sistema nãoreinalatório duplo $\mathrm{T}$ de Baraka, em uma freqüência respiratória de 10 a 15 movimentos por minuto. Como foi possível visualizar os pulmões durante a maior parte do tempo anestésico-cirúrgico, a pressão manual exercida foi aquela que permitisse a completa expansão pulmonar, sem interferir com o ato operatório. Não foi instituída ventilação mecânica porque inviabilizaria o desenvolvimento da técnica cirúrgica. Os animais foram submetidos à toracotomia intercostal para implante de um segmento autólogo de pericárdio no diafragma.

Foram avaliadas as seguintes variáveis: freqüência cardíaca (oximetria de pulso e/ou auscultação esofágica), saturação de oxigênio da hemoglobina (oximetria de pulso ${ }^{\mathrm{h}}$ ), pressão arterial sistólica (método não invasivo com doppler ultrassônicoi) e vaporização do halotano $(\mathrm{V} \%)$. As variáveis foram mensuradas 20 minutos após a MPA (TMPA) e 10 minutos após indução e a cada 10 minutos até o final do procedimento cirúrgico (T10 a T100). 
O grau de sedação após MPA e a qualidade da recuperação anestésica foram observadas. O grau de sedação foi avaliado através da seguinte escala: bem alerta, reage quando estimulado, não reage quando estimulado e sedado. Quando à qualidade da recuperação observaram-se os parâmetros a seguir: a recuperação foi classificada como de boa qualidade quando ocorreu de uma a duas tentativas do animal de posicionar-se em pé com sucesso, sem presença de agitação, excitação, movimentos de pedalagem e vocalização; dita satisfatória quando ocorreu de duas a cinco tentativas de posicionar-se em pé com sucesso, movimentos de pedalagem presentes e vocalização; e recuperação pobre quando os animais apresentarem movimentos de pedalagem (ataxia), agitação, vocalização, excitação e defecação.

A necessidade ou não de butorfanol $(0,1 \mathrm{mg}$ $\left.\mathrm{kg}^{-1} \mathrm{IV}\right)$ e atropina $\left(0,022 \mathrm{mg} \mathrm{kg}^{-1} \mathrm{IV}\right)$ no transcirúrgico também foi avaliada. Para administração de doses adicionais de butorfanol no transcirúrgico observouse o aumento do tono mandibular, aumento de 10 a $20 \%$ da freqüência cardíaca e pressão arterial sistólica, aumento do reflexo palpebral e perda do relaxamento muscular na área operatória (informada pelo cirurgião). Administrou-se atropina durante o transoperatório sempre que os valores de freqüência cardíaca se apresentavam abaixo de 100bpm.

Os resultados obtidos foram comparados estatisticamente através de análise de variância (ANOVA), e comparação de médias pelo teste de Bonferroni. O grau de significância estabelecido foi de $5 \%(\mathrm{p}<0,05)$.

\section{RESULTADOS}

A freqüência cardíaca reduziu significativamente em relação ao TMPA, nos tempos 10, 20, 60, 80, 90 e 100 minutos (Tabela 1). $O$ maior decréscimo na freqüência cardíaca ocorreu aos 100 minutos $(134,0 \pm 6,76)$. Em todos os tempos, a freqüência cardíaca manteve-se dentro da faixa de variação fisiológica para gatos.

A pressão arterial sistólica reduziu significativamente em relação ao TMPA, dos 10 aos 90 minutos, com máximo de decréscimo aos 30 minutos $(94,1 \pm 26,3)$. Aos 100 minutos, a pressão sistólica aumentou não diferindo estatisticamente em relação ao TMPA (Tabela 1).

A saturação de oxigênio da hemoglobina manteve-se ao redor de $100 \%$ em todos os tempos (Tabela 1). Houve redução estatisticamente significativa na vaporização do halotano a partir de T30 até final da cirurgia
(Tabela 1). Aos 10 minutos após indução anestésica, a vaporização encontrava-se ao redor de $0,85 \% \pm 0,11$ reduzindo-se gradativamente, aos 70 minutos $(0,33 \%$ $\pm 0,04)$ e aos 100 minutos $(0,16 \% \pm 0,06)$.

Aos 20 minutos após MPA, todos os animais encontravam-se em estado alerta, sem sinais clínicos de sedação. Todos os animais apresentaram recuperação anestésica tranqüila, sem excitação e com uma ou duas tentativas os animais posicionavam-se em pé com sucesso.

Em nenhum dos animais foi necessária a administração de dose trans-operatória de butorfanol. Em dois animais, foi administrado sulfato de atropina $\left(0,022 \mathrm{mg} \mathrm{kg}^{-1} \mathrm{IV}\right)$ no transcirúrgico quando a freqüência cardíaca reduziu-se a valores inferiores a $100 \mathrm{bpm}$.

\section{DISCUSSÃO}

O butorfanol é considerado um analgésico eficaz para gatos em procedimentos que induzam dor leve à moderada, especialmente dor visceral (WAGNER, 1999). Em humanos, o tramadol é utilizado para tratamento de dor moderada a severa (LEE et al., 1993).

Neste estudo, a associação de um opióide agonista-antagonista, o butorfanol, e de um opióide 'atípico', o tramadol, no período pré-operatório, promoveu analgesia satisfatória para realização de toracotomia intercostal em gatos. A toracotomia é

Tabela 1 - Valores médios e erro padrão da média da vaporização de halotano $(\mathrm{V} \%)$, freqüência cardíaca (FC batimentos/minuto), pressão arterial sistólica (PAS $-\mathrm{mmHg}$ ) e saturação de oxigênio na hemoglobina $\left(\mathrm{SPO}_{2}-\%\right)$ nos diversos tempos de avaliação.

\begin{tabular}{lcccc}
\hline \multirow{2}{*}{$\begin{array}{l}\text { Tempo } \\
(\mathrm{min})\end{array}$} & \multicolumn{4}{c}{ Variáveis } \\
\cline { 2 - 5 } & $\mathrm{V} \%$ & $\mathrm{FC}(\mathrm{bpm})$ & $\mathrm{PAS}(\mathrm{mmHg})$ & $\mathrm{SPO}_{2}(\%)$ \\
\hline TMPA & $0,00 \pm 0,00$ & $175,5 \pm 10,16$ & $123,8 \pm 8,71$ & ----- \\
10 & $0,85 \pm 0,11$ & $142,4 \pm 6,72^{*}$ & $87,5 \pm 4,18^{*}$ & $99,9 \pm 0.08$ \\
20 & $0,67 \pm 0,06$ & $141,3 \pm 4,91^{*}$ & $97,5 \pm 5,37^{*}$ & $99,8 \pm 0,13$ \\
30 & $0,60 \pm 0,06^{*}$ & $154,3 \pm 6,52$ & $94,1 \pm 7,59^{*}$ & $99,8 \pm 0,18$ \\
40 & $0,44 \pm 0,07^{*}$ & $154,0 \pm 5,33$ & $94,4 \pm 8,69^{*}$ & $99,5 \pm 0,26$ \\
50 & $0,35 \pm 0,05^{*}$ & $156,0 \pm 5,95$ & $97,4 \pm 6,68^{*}$ & $99,8 \pm 0,18$ \\
60 & $0,33 \pm 0,04^{*}$ & $152,8 \pm 6,54^{*}$ & $100,7 \pm 4,44^{*}$ & $99,8 \pm 0,11$ \\
70 & $0,33 \pm 0,04^{*}$ & $154,3 \pm 6,34$ & $97,5 \pm 5,28^{*}$ & $99,7 \pm 0,19$ \\
80 & $0,26 \pm 0,06^{*}$ & $146,3 \pm 7,12^{*}$ & $98,5 \pm 6,70^{*}$ & $99,6 \pm 0,19$ \\
90 & $0,22 \pm 0,06^{*}$ & $142,3 \pm 7,33^{*}$ & $101,9 \pm 6,50^{*}$ & $99,9 \pm 0,11$ \\
100 & $0,16 \pm 0,06^{*}$ & $134,0 \pm 6,76^{*}$ & $106,4 \pm 8,22$ & $99,8 \pm 0,16$ \\
\hline
\end{tabular}

*Diferença estatisticamente significativa $(\mathrm{p}<0,05)$ em relação ao TMPA, exceto $\mathrm{V} \%$ e $\mathrm{SPO}_{2} \%$ em relação a $\mathrm{T} 10$.

Ciência Rural, v. 33, n. 5, set-out, 2003. 
considerada um procedimento de dor severa (HELLYER, 1999).

Este efeito analgésico preventivo pode ser comprovado pela baixa vaporização de halotano durante o procedimento cirúrgico, permancendo a partir de T20 abaixo da concentração alveolar mínima para gatos que varia de 0,8 a 1,2 V\% (PADDLEFORD, 1999).

O tramadol produz analgesia mediada por receptores opióides $m u$ e via de ação não opióide, a inibição da recaptação de monoaminas (RAFFA et al., 1992). A afinidade do tramadol por receptores $m u$ é aproximadamente $6000 \mathrm{vez}$ menor que a da morfina (DAYER et al., 1997). Devido à afinidade do tramadol por receptores opióides $m u$ ser relativamente fraca (RAFFA et al., 1992), o butorfanol, com sua atividade antagonista de receptores $m u$, poderia bloquear esta via de antinocicepção do tramadol. Os agonistasantagonistas, como butorfanol apresentam grande afinidade por seus receptores, maior do que agonistas puros, assim, quando administrados concomitantemente, eles deslocam os agonistas puros de seus receptores (JOHNSON, 1991).

Desta forma, a analgesia promovida pela associação tramadol-butorfanol deve ocorrer pela inibição da recaptação de norepinefrina e serotonina promovida pelo tramadol e pela ativação de receptores kappa pelo butorfanol.

Quando se administra naloxone após tramadol, a ação analgésica deste é somente parcialmente inibida, confirmando a existência de outra via de antinocicepção, além da mediada por receptores $m u$ opióides (DAYER et al., 1997).

O butorfanol por ser um opióide agonistaantagonista, com atividade agonista sobre receptores kappa, produz nas doses de 0,1 a $0,8 \mathrm{mg} \mathrm{kg}^{-1}$ mínima depressão cardiovascular e respiratória (WERNER \& TABOADA, 1994). O tramadol, diferindo de outros opióides agonista $m u$, não produz alterações relevantes no sistema respiratório e cardiovascular (SCOTT \& PERRY, 2000).

Em gatos, o uso intravenoso ou de altas doses de butorfanol pode desencadear hipotensão e bradicardia transitória (WERNER \& TABOADA, 1994). Com o intuito de prevenir este efeito, administrou-se sulfato de atropina, concomitantemente, à associação tramadol/ butorfanol.

Neste estudo, a associação butorfanol e tramadol produziu redução estatisticamente significativa da freqüência cardíaca e pressão arterial sistólica em relação aos valores TMPA, porém estas variáveis mantiveram-se dentro da faixa de variação fisiológica para gatos, não caracterizando bradicardia, nem hipotensão. MUIR et al. (2000) consideram a faixa de variação fisiológica da freqüência cardíaca para gatos de 145 a 200bpm, caracterizando como bradicardia valores inferiores a $100 \mathrm{bpm}$. Observouse aos 100 minutos o valor mais baixo de freqüência cardíaca $(134,0 \pm 6,76)$.

HASKINS (1996) considera faixa de variação fisiológica da pressão sistólica para cães e gatos, valores de 100 a $160 \mathrm{mmHg}$, e caracteriza hipotensão valores abaixo de $80 \mathrm{mmHg}$. Neste estudo, em nenhum dos tempos os animais apresentaram valores de pressão arterial sistólica abaixo de 80 mmHg. Aos 10 minutos após indução, teve-se o valor mais baixo de pressão sistólica $(87,5 \pm 4,18)$, sendo que houve aumento gradativo da pressão arterial no decorrer no período transcirúrgico. Acredita-se que o agente indutor, o propofol, seja o responsável pela queda brusca da pressão arterial sistólica logo após indução. As alterações cardiovasculares induzidas pela administração de propofol consistem principalmente de decréscimo na pressão sanguínea arterial sistólica, média e diastólica (SHORT \& BUFALARI, 1999).

Não se observou sedação após a administração da associação tramadol/butorfanol/ atropina. Segundo WERNER \& TABOADA (1994) o butorfanol não produz sedação em gatos. SAWYER \& RECH (1987) observaram, além de mínima sedação, midríase e apreensão em alguns gatos. O tramadol em humanos produz sedação (SCOTT \& PERRY, 2000). O efeito sedativo do tramadol deve ocorrer via receptor opióide $m u$, porém nesse experimento esta via, provavelmente, está bloqueada pelo butorfanol.

\section{CONCLUSÃO}

A associação tramadol $\left(2,0 \mathrm{mg} \mathrm{kg}^{-1}\right)$, butorfanol $\left(0,4 \mathrm{mg} \mathrm{kg}^{-1}\right)$ e atropina $\left(0,044 \mathrm{mg} \mathrm{kg}^{-1}\right)$, via intramuscular, na pré-medicação de gatos anestesiados com propofol ( $\left.5,0 \mathrm{mg} \mathrm{kg}^{-1}\right)$ e halotano, produz mínimas alterações cardiovasculares, não induz sedação e promove analgesia satisfatória para realização de toracotomia intercostal lateral em gatos.

\section{FONTES DE AQUISIÇÃO}

a - Tramal 100mg/2ml - Monsanto do Brasil Ltda. - São Paulo, $\mathrm{SP}-$ Brasil.

b - Stadol 2mg/ml - Apothecon - USA

c - Sulfato de Atropina $0,5 \mathrm{mg} / \mathrm{ml}$ - Santisa Laboratório Farmacêutico - Bauru, SP - Brasil.

d - Propovan 10mg/ml - Cristália - Produtos Químicos Farmacêuticos Ltda. - Itapira, SP - Brasil.

e - Halotano - Cristália - Produtos Químicos Farmacêuticos Ltda. - Itapira, SP - Brasil. 
$\mathrm{f}-$ Xylestesin $2 \%$ sem vasoconstritor - Cristália - Produtos Químicos Farmacêuticos Ltda. - Itapira, SP - Brasil.

$\mathrm{g}$ - Ketofen 1\% - Rhodia-Mérieux Veterinária Ltda. - Paulina, SP - Brasil.

h - Pulse Oximeter - Nellcor model M-200-13 - USA.

i - Pocket Doppler - model 841 - A - Parks Medical Electronics.

j - Vaporizador Calibrado Halothane HB 4.2

\section{REFERÊNCIAS BIBLIOGRÁFICAS}

DAYER P.; DESMEULES J.; COLLART L. Pharmacology of tramadol. Drugs, v.53, s.2, p.18-24, 1997.

HASKINS S.C. Monitoring the anesthetized patient. In: LUMB W.V.; JONES E.W. Veterinary anesthesia. 3.ed. Baltimore : Williams \& Wilkins, 1996. Cap.15, p.409-424.

HELLYER P.W. Minimizing postoperative discomfort in dogs and cats. Veterinary Medicine, n.3, p.259-265, 1999.

HELLYER P.W.; GAYNOR J.S. Acute postsurgical pain in dogs and cats. Compendium on Continuing Education for the Practicing Veterinarian - small animal, v.20, n.2, p.140-153, 1998.

JOHNSON J.M. The veterinarian's responsibility: assessing and managing acute pain in dogs and cats. Part I and II. Compendium on Continuing Education for the Practicing Veterinariansmall animal, v.13, n.5/6, p.804-807; 911-916, 1991.

LEE C.R.; McTAVISH D.; SORKIN E.M. Tramadol - a preliminary review of its pharmacodynamic and pharmacokinetic properties, and therapeutic potential in acute and chronic pain states. Drugs, v.46, n.2, p.313-340, 1993.
MUIR W.W. et al. Patient monitoring during anesthesia. In: Handbook of veterinary anesthesia. 3.ed. Missouri : Mosby, 2000. Cap.16, p.250-283.

ORSINI J.A. Butorphanol tartrate: pharmacology and clinical indications. Compendium on Continuing Education for the Practicing Veterinarian - equine, v.10, n.7, p.849-854, 1988.

PADDLEFORD R.R. Anesthetic agents. In: Manual of small animal anesthesia. Philadelphia : Saunders, 1999. Cap.3, p.31-77.

RAFFA R.B. et al. Opioid and nonopioid components independently contribute to the mechanism of action of tramadol, an "atypical" opioid analgesic. The Journal of Pharmacology and Experimental Therapeutics, v.260, n.1, p.275-285, 1992.

SAWYER D.C.; RECH R.H. Analgesia and behavioral effects of butorphanol, nalbuphine, and pentazocine in the cat. Journal of the American Animal Hospital Association, v.23, p.438-446, 1987.

SCOTT L.J.; PERRY, C.M. Tramadol: a review of its use in perioperative pain. Drugs, v.60, n.1, p.139-176, 2000.

SHORT C.E.; BUFALARI, A. Propofol anesthesia. Veterinary Clinical of north America small animal Practice, v.29, n.3, p.747-748, 1999.

WAGNER, A.E. Is butorphanol analgesic in dogs and cats? Veterinary Medicine, n.4, p.346-350, 1999.

WERNER B.E.; TABOADA J. Use of analgesics in feline medicine. Compendium on Continuing Education for the Practicing Veterinarian - small animal, n.4, p.493-499, 1994. 